\title{
Thrombolysis in acute ischemic stroke in patients with dementia
}

\author{
A Swedish registry study \\ OPEN
}

Eva Zupanic, MD

Mia von Euler, MD, PhD

Ingemar Kåreholt, $\mathrm{PhD}$

Beatriz Contreras

Escamez, MD

Johan Fastbom, MD,

$\mathrm{PhD}$

Bo Norrving, MD, PhD

Dorota Religa, MD, PhD

Milica G. Kramberger,

MD, PhD

Bengt Winblad, MD,

$\mathrm{PhD}$

Kristina Johnell, $\mathrm{PhD}$

Maria Eriksdotter, MD,

$\mathrm{PhD}$

Sara Garcia-Ptacek, MD, $\mathrm{PhD}$

Correspondence to

Dr. Garcia-Ptacek:

sara.garcia-ptacek@ki.se

\section{ABSTRACT}

Objective: To compare access to intravenous thrombolysis (IVT) for acute ischemic stroke (AIS) and its outcomes in patients with and without dementia.

Methods: This was a longitudinal cohort study of the Swedish dementia and stroke registries. Patients with preexisting dementia who had AIS from 2010 to 2014 ( $n=1,356)$ were compared with matched patients without dementia $(n=6,755)$. We examined access to thrombolysis and its outcomes at 3 months (death, residency, and modified Rankin Scale [mRS] score). Odds ratios (ORs) and 95\% confidence intervals (Cls) were calculated with logistic and ordinal logistic regression.

Results: The median age at stroke onset was 83 years in both groups. IVT was administered to 94 (7.0\%) patients with dementia and 639 (9.5\%) patients without dementia. The OR of receiving IVT was 0.68 (95\% Cl 0.54-0.86) for patients with dementia. When the analysis was repeated exclusively among patients independent in everyday activities, dementia status was no longer significant (OR 0.79, 95\% Cl 0.60-1.06). However, differences persisted in patients $\leq 80$ years of age (OR $0.58,95 \% \mathrm{Cl} 0.36-0.94)$. In patients who received thrombolysis, the incidence of symptomatic intracerebral hemorrhage $\left(\mathrm{sICH}_{;} 7.4 \%\right.$ vs $\left.7.3 \%\right)$ and death at 3 months $(22.0 \% \mathrm{vs}$ $18.8 \%)$ did not differ significantly between the 2 groups. However, mRS score and accommodation status were worse among patients with dementia after 3 months in adjusted analyses (both $p<0.001$ ). Unfavorable outcomes with an mRS score of 5 to 6 were doubled in patients with dementia (56.1\% vs $28.1 \%)$.

Conclusions: Younger patients with dementia and AIS are less likely to receive IVT. Among patients receiving thrombolysis, there are no differences in $\mathrm{s} \mathrm{CH}$ or death, although patients with dementia have worse accommodation and functional outcomes at 3 months. Neurology ${ }^{\circledR}$ 2017;89:1860-1868

\section{GLOSSARY}

ADL = activities of daily living; AIS $=$ acute ischemic stroke; $\mathbf{A T C}=$ Anatomical Therapeutic Chemical; $\mathbf{C l}=$ confidence interval; ICD-10 = International Classification of Diseases, 10th revision; IVT = intravenous thrombolysis; MMSE = MiniMental State Examination; $\mathbf{m R S}=$ modified Rankin Scale; NIHSS = NIH Stroke Scale; OR = odds ratio; Riksstroke = Swedish national quality registry for acute vascular diseases of the brain; $\mathbf{R L S}=$ Reaction Level Scale; $\mathbf{s} \mathbf{I C H}=$ symptomatic intracranial hemorrhage; SveDem = Swedish Dementia Registry

Dementia is not a contraindication for intravenous thrombolysis (IVT) in acute ischemic stroke (AIS), but most of the thrombolysis studies to date excluded or underrepresented octogenarians and nonagenarians, ${ }^{1,2}$ and guidelines differ in their recommendations for IVT use in this population. According to the American Heart Association/American Stroke Association,

From the Karolinska Institutet (E.Z., D.R., B.W.), Department of Neurobiology, Care Sciences and Society, Center for Alzheimer Research, Division of Neurogeriatrics, Huddinge, Sweden; Department of Neurology (E.Z., M.G.K.), University Medical Centre, Ljubljana, Slovenia; Karolinska Institutet (M.v.E.), Department of Clinical Science and Education, Södersjukhuset, and Department of Medicine, Solna; Karolinska University Hospital (M.v.E.), Department of Clinical Pharmacology; Karolinska Institutet and Stockholm University (I.K., J.F., K.J.), Aging Research Center, Stockholm, Sweden; Jönköping University (I.K.), Institute of Gerontology, School of Health and Welfare, Aging Research Network-Jönköping; Karolinska Institutet (B.C.E., M.E., S.G.-P.), Department of Neurobiology, Care Sciences and Society, Center for Alzheimer Research, Division of Clinical Geriatrics, Huddinge, Sweden; Department of Geriatrics (B.C.E.), Hospital Universitario de Getafe, Madrid, Spain; Lund University (B.N.), Skane University Hospital, Department of Clinical Sciences Lund, Neurology; Karolinska University Hospital, Department of Geriatric Medicine (D.R., M.E., S.G.-P.); and Södersjukhuset (S.G.-P.), Department of Internal Medicine, Section for Neurology, Stockholm, Sweden.

Go to Neurology.org for full disclosures. Funding information and disclosures deemed relevant by the authors, if any, are provided at the end of the article. The Article Processing Charge was funded by Swedish Research Council and Johanniterorden i Sverige/Swedish Order of Saint John.

This is an open access article distributed under the terms of the Creative Commons Attribution License 4.0 (CC BY), which permits unrestricted use, distribution, and reproduction in any medium, provided the original work is properly cited. 
patients with dementia may benefit from IVT; however, decisions should be made individually with premorbid functional level taken into account. ${ }^{3}$ Alteplase is not indicated in patients $>80$ years of age according to the European Medicines Agency, ${ }^{4}$ while it is recommended in selected patients according to European stroke guidelines. ${ }^{5}$ Because of this inconsistency and fear of cerebral hemorrhage, physicians may adopt a more conservative approach in patients with dementia. ${ }^{2,6}$ Indeed, patients with dementia had increased mortality and poorer functional outcomes after stroke regardless of the use of reperfusion therapy in some $e^{7,8}$ but not other studies. ${ }^{9,10}$ Patients with dementia are less likely to receive IVT, ${ }^{8,10}$ even though increased incidence of symptomatic intracranial hemorrhage $(\mathrm{sICH})$ has so far not been reported. ${ }^{7,9,10}$

There is a lack of recent data on use and outcomes of IVT in patients with dementia, and this subject has been identified as a high-priority research area. ${ }^{3}$ Our aim is to analyze the use and outcomes of IVT for AIS in patients with preexisting dementia in a large national cohort to determine whether dementia status is associated with lower use or poorer outcomes after IVT.

METHODS Quality registries and study population. We performed a longitudinal cohort study of patients diagnosed with dementia who subsequently had a first AIS. Patients with dementia were identified from Swedish Dementia Registry (SveDem), which has previously been described. ${ }^{11,12}$ Registration occurs at the time of dementia diagnosis with information on dementia type, demographics, and living situation.

Occurrence of AIS was identified with Riksstroke, the Swedish national quality registry for acute vascular diseases of the brain, one of the world's largest stroke registries. ${ }^{13}$ All hospitals admitting acute stroke patients participate, and coverage for ischemic stroke is $>90 \% .{ }^{14}$ Information on the registries is available at svedem.se and riksstroke.org.

Data on medication therapy from 2005 were obtained from the Swedish Prescribed Drug Register, including all prescription medications dispensed at Swedish pharmacies, with coverage of $\approx 100 \%{ }^{15}$ Comorbidities were collected from the Swedish National Inpatient Register, available from 1998 and coded according to the ICD-10, at present covering all in-hospital and specialist clinic diagnoses. ${ }^{16}$

Patients with a dementia diagnosis who subsequently had AIS were selected and matched by age ( \pm 3 years), sex, year of stroke, and geographic region with controls without dementia from Riksstroke. Controls without dementia were excluded if they ever had a SveDem registration, ever were diagnosed with dementia or confusional syndrome (ICD-10 code F00-F09 or G30-G32), or ever had taken antidementia medication (Anatomical Therapeutic Chemical [ATC] codes N06DX and
N06DA, including donepezil, rivastigmine, galantamine, and memantine).

Of 58,154 patients registered in SveDem between May 2007 and December 2014, a total of 2,233 patients with dementia subsequently had AIS and were matched to 8,963 dementia-free controls from Riksstroke. The patient selection process is illustrated in the figure. Because of the changes in the IVT treatment window to 4.5 hours in 2009, data from 2010 to 2014 were used, and a study population of 1,356 AIS patients with dementia and 6,755 AIS controls without dementia was available for analyses.

Variables. From SveDem, we used information on dementia type, date, and cognitive evaluation with the Mini-Mental State Examination (MMSE).

From Riksstroke, we used data on stroke event, demographics, living arrangements, transport to the hospital, activation of stroke code (protocol for management of acute stroke), IVT treatment, symptom-to-needle time (time interval between the symptom onset and initiation of the IVT), follow-up, and death. Independence in activities of daily living (ADL) before and after stroke was defined as independence in mobility, dressing, and toileting. Urban and rural typology was defined for Swedish county councils ("landsting") on the basis of Organisation for Economic Co-operation and Development methodology. ${ }^{17}$ We used 2 clinical assessment tools as proxies for stroke severity: level of consciousness at admission to the hospital, determined with Reaction Level Scale (RLS), in which patients with RLS score of 1 are defined as alert, those with RLS score of 2 to 3 are defined as lethargic, and those with RLS score of 4 to 8 are defined as unconscious, and the NIH Stroke Scale (NIHSS). sICH is defined in Riksstroke as clinical worsening with an increase of $\geq 4$ NIHSS points in the presence of intracranial hemorrhage starting $<36$ hours after the start of thrombolysis (nonsymptomatic intracranial hemorrhage is not recorded). Modified Rankin Scale (mRS) is a scale measuring degree of disability. Because $\mathrm{mRS}$ is a standard assessment method in stroke research and is missing in the Riksstroke registry, mRS score was estimated by translation of 5 self-reported Riksstroke functional outcome variables. This previously described conversion method offers high precision but cannot differentiate between functional groups with an mRS score of 0 to $2 .{ }^{18}$ Conversion was possible for mRS only after (and not before) stroke.

Number of medications was defined as the sum of all prescription drugs dispensed from pharmacies during the 3 months preceding the stroke, obtained from the Swedish Prescribed Drug Register, and was used as a proxy for comorbidity. ${ }^{12}$

Statistical analysis. For categorical variables, data are presented as number of cases and percentages. Continuous variables were summarized as mean $\pm \mathrm{SD}$ or median (interquartile range). For calculating significant differences, the Student $t$ test and Mann-Whitney $U$ test were used for continuous and $\chi^{2}$ or Fisher exact tests for categorical variables.

Multivariate logistic regression analyses were used to assess the relationship between dementia status and receiving IVT. We assessed different outcomes after IVT: using multivariate logistic regression for (1) death at 3 months, (2) nursing home placement at 3 months, and (3) mRS score of 4 or 5 (vs mRS score $<4)$ at 3 months and using multivariate ordinal logistic regression for (4) mRS score at 3 months as an ordinal scale of step-wise increase in mRS score. For mRS score, we tested the proportional odds/parallel-lines assumption using the STATA command GOLOGIT2 with a gamma parameterization. No significant violations of the assumptions were found.

Initial regression models were adjusted for age and sex. Next, we tested contraindications to IVT, possible confounding living 


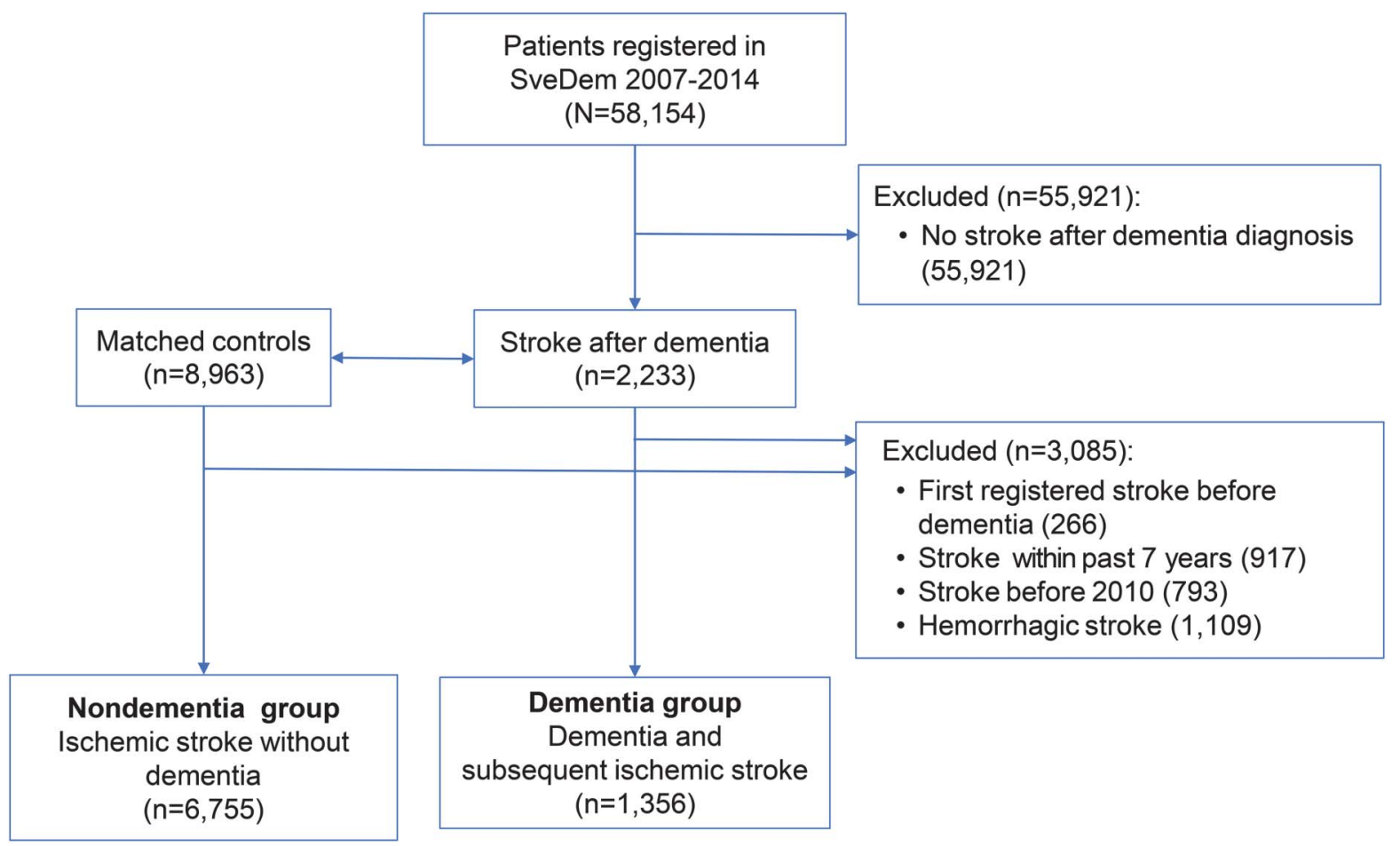

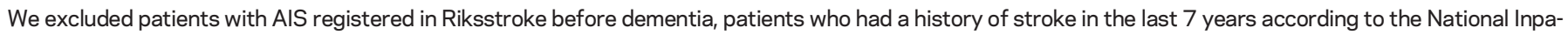

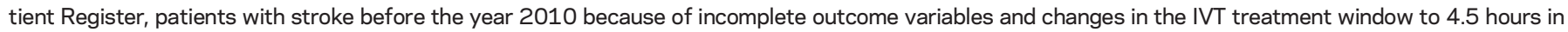

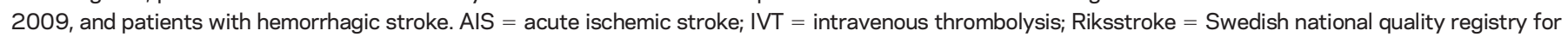
acute vascular diseases of the brain; SveDem = Swedish Dementia Registry.

arrangements, comorbidities, and medication, adding covariates in a stepwise manner. Covariates were chosen to reflect a burden of comorbidity, which might affect the decision to thrombolyse or, in the case of living situation, could affect time to hospital arrival and obtaining information. Individual drugs were subtracted from total number of medications if also used as separate confounders. We tested several interactions between comorbidities and medication. We tested all variables with $p \leq 0.25$ significance in the univariate models. Variables that did not reach the significance of $p<0.05$ in a multivariate model, did not change the $\beta$ coefficient by $\approx 20 \%$, or did not improve the $R^{2}$ of the model were discarded. Adjusted odds ratios (ORs) with 95\% confidence intervals (CIs) are presented. Post hoc analyses including NIHSS are also shown.

Post hoc propensity score-adjusted models were conducted. The propensity scores were obtained from multiple logistic regression for dementia status including the variables age, sex, smoking, number of medication, antiaggregants, antipsychotics, antidepressants, lipid-lowering agents, antihypertensives, atrial fibrillation, diabetes mellitus, hip fracture, ischemic heart disease, heart failure, renal failure, and liver failure (variables as shown in table 1).

Tests were 2 tailed with a value of $p<0.05$ considered significant. The IBM Statistical Package for Social Sciences for Windows, Sciences Software version 23 (IBM Corp, Armonk, NY) and STATA version 14 (StataCorp, College Station, TX) were used.

Standard protocol approvals, registrations, and patient consents. This study complies with the Declaration of Helsinki and was approved by the regional ethics review board in Stockholm, Sweden (dnr 2015/743-31/4). Patients and relatives were informed of inclusion in the registries at the time of diagnosis and could decline participation or withdraw consent. Data were deidentified before analysis.

RESULTS Characteristics of the study population. Of 8,111 patients with AIS, 1,356 patients had preexisting dementia and 6,755 patients did not have dementia registered. Because national Swedish stroke guidelines did not recommend IVT in patients $>80$ years of age until 2014 (except in particular cases after careful consideration), ${ }^{19}$ we age-stratified the cohort (table 1 ).

In the dementia group, at the time of the registration in SveDem, median MMSE score was 21 (5), which is in line with previous SveDem studies. ${ }^{12,20}$ Median time from dementia diagnosis to stroke was 546 days (705 days), and total days at risk were 894,439. Alzheimer dementia and mixed dementia were the most common dementia types ( $\mathrm{n}=628$, $46.3 \%)$, and 311 patients $(22.9 \%)$ had vascular dementia (results not presented).

Use of thrombolysis. Of all 8,111 patients, 733 (9.0\%) received IVT, 94 (7.0\%) patients with dementia and 639 (9.5\%) patients without dementia. Patients with dementia were less often independent before stroke (52.8\% vs $87.1 \%, p<0.001)$. Patients who received IVT were more often independent than their 
counterparts who did not receive IVT $(90.5 \%$ ADL independence in IVT group vs $80.7 \%$ in non-IVT group, $p<0.001$; results not presented).

Patients with dementia treated with IVT were older (median age 83 vs 81 years, $p=0.016$ ) and received more medications (median 6 vs $4, p<0.001$ ) than IVT-treated controls. Apart from ischemic heart disease, there were no other important differences in comorbidities. Other characteristics are shown in table 2. There were no differences between the 2 thrombolysis

\begin{tabular}{|c|c|c|c|c|c|c|}
\hline \multirow[t]{3}{*}{ Table 1} & nd comorbiditi & efore stroke in pa & its with $A$ & with and withou & ementia, stratified & age \\
\hline & \multicolumn{3}{|c|}{ Patients $\leq 80$ y of age $(n=2841)$} & \multicolumn{3}{|c|}{ Patients $>80$ y of age $(n=5270)$} \\
\hline & $\begin{array}{l}\text { With dementia } \\
(n=436)\end{array}$ & $\begin{array}{l}\text { Without dementia } \\
(n=2,405)\end{array}$ & $p$ Value & $\begin{array}{l}\text { With dementia } \\
(\mathrm{n}=920)\end{array}$ & $\begin{array}{l}\text { Without dementia } \\
(n=4,350)\end{array}$ & $p$ Value $^{a}$ \\
\hline Age at first stroke, median (IQR), y & 77 (6) & $76(7)$ & 0.190 & $86(6)$ & $86(6)$ & 0.871 \\
\hline Female sex, $n(\%)$ & $197(45.2)$ & $1,124(46.7)$ & 0.550 & 567 (61.6) & $2,714(62.4)$ & 0.666 \\
\hline Nursing home placement, $n$ (\%) & 96 (22.3) & $63(2.6)$ & $<0.001$ & $327(35.8)$ & 441 (10.2) & $<0.001$ \\
\hline Lives alone (not in a nursing home), $\mathrm{n}(\%)^{\mathrm{a}}$ & $119(27.7)$ & $896(37.5)$ & $<0.001$ & 327 (35.9) & $2,485(57.4)$ & $<0.001$ \\
\hline ADL independent, $n(\%)^{a}$ & $262(62.7)$ & $2,237(93.7)$ & $<0.001$ & $412(48.0)$ & $3,548(83.4)$ & $<0.001$ \\
\hline Smoking, $\mathrm{n}(\%)^{\mathrm{a}}$ & 29 (7.3) & $320(14.4)$ & 0.001 & $25(3.0)$ & $194(4.9)$ & 0.018 \\
\hline Medications, n, median (IQR) & $6(5)$ & $4(5)$ & $<0.001$ & $6(5)$ & $5(5)$ & $<0.001$ \\
\hline Antiaggregants, $n(\%)$ & $181(41.5)$ & $732(30.4)$ & $<0.001$ & $460(50.0)$ & $1,768(40.6)$ & $<0.001$ \\
\hline Antipsychotics, n (\%) & $30(6.9)$ & 32 (1.3) & $<0.001$ & $71(7.7)$ & $62(1.4)$ & $<0.001$ \\
\hline Antidepressants, $\mathrm{n}(\%)$ & 154 (35.3) & $214(8.9)$ & $<0.001$ & $272(29.6)$ & $509(11.7)$ & $<0.001$ \\
\hline Antihypertensives, n (\%) & $272(62.4)$ & $1,460(60.7)$ & 0.509 & $620(67.4)$ & $3,080(70.8)$ & 0.040 \\
\hline Antidiabetics, n (\%) & $90(20.6)$ & 367 (15.3) & 0.005 & 94 (10.2) & $483(11.1)$ & 0.434 \\
\hline Statins, n (\%) & $107(24.5)$ & $543(22.6)$ & 0.369 & $139(15.1)$ & 797 (18.3) & 0.021 \\
\hline AF, n (\%) & $108(24.8)$ & $449(18.7)$ & 0.003 & 345 (37.5) & 1,425 (32.8) & 0.006 \\
\hline Warfarin in patients with $\mathrm{AF}, \mathrm{n}(\%)$ & 24 (22.2) & $101(22.5)$ & 0.951 & $29(8.4)$ & $241(16.9)$ & $<0.001$ \\
\hline Diabetes mellitus, n (\%) & $108(24.8)$ & 445 (18.5) & 0.002 & 165 (17.9) & $729(16.8)$ & 0.388 \\
\hline Hypertension, n (\%) & $222(50.9)$ & $1,079(44.9)$ & 0.020 & $552(60.0)$ & 2,402 (55.2) & 0.008 \\
\hline Femur fracture, $n(\%)$ & $41(9.4)$ & $90(3.7)$ & $<0.001$ & $133(14.5)$ & $465(10.7)$ & 0.001 \\
\hline Any hemorrhage, $n$ (\%) & $61(14.0)$ & $210(8.7)$ & 0.001 & $126(13.7)$ & 479 (11.0) & 0.020 \\
\hline Ischemic heart disease, n (\%) & $105(24.1)$ & $230(22.0)$ & 0.346 & $267(29.0)$ & 1,312 (30.2) & 0.493 \\
\hline Heart failure, n (\%) & 69 (15.8) & 277 (11.5) & 0.011 & 207 (22.5) & $1,001(23.0)$ & 0.737 \\
\hline Renal disease, n (\%) & $43(9.9)$ & $157(6.5)$ & 0.012 & $86(9.3)$ & $448(10.3)$ & 0.385 \\
\hline Liver disease, n (\%) & $6(1.4)$ & $43(1.8)$ & 0.543 & $15(1.6)$ & $41(0.9)$ & 0.064 \\
\hline Stroke code, $\mathrm{n}(\%)^{\mathrm{b}}$ & $94(22.1)$ & $655(27.7)$ & 0.016 & $244(27.1)$ & $1,080(25.3)$ & 0.242 \\
\hline Arrival by ambulance, $n(\%)^{\mathrm{b}}$ & $294(82.1)$ & $1,314(68.3)$ & $<0.001$ & $710(92.3)$ & 2,992 (81.7) & $<0.001$ \\
\hline Unconscious at arrival, $n(\%)^{b}$ & $12(2.8)$ & $61(2.6)$ & 0.778 & $40(4.4)$ & $177(4.1)$ & 0.671 \\
\hline Unconscious or lethargic (RLS>1), $n(\%)^{b}$ & 78 (18.2) & 221 (9.3) & $<0.001$ & $215(23.8)$ & 788 (18.3) & $<0.001$ \\
\hline Thrombolysis, $n(\%)^{b}$ & $29(6.7)$ & 282 (11.8) & 0.002 & $65(7.1)$ & 357 (8.2) & 0.255 \\
\hline
\end{tabular}

Abbreviations: $\mathrm{ADL}$ = activities of daily living; $\mathrm{AF}$ = atrial fibrillation; $\mathrm{AIS}=$ acute ischemic stroke; IQR = interquartile range; IVT = intravenous thrombolysis; RLS = Reaction Level Scale.

Antiaggregants (acetylsalicylic acid and clopidogrel) $=$ Anatomical Therapeutic Chemical (ATC) codes B01AC06 and B01AC04; antipsychotics $=$ ATC code N05A; antidepressants = ATC code N06A; antihypertensives = ATC codes CO2-CO9D; drugs in diabetes mellitus = ATC code A10; statins = ATC code C10AA; and warfarin $=$ ATC code B01AA03.

Number of medications refers to all prescribed medication; unconscious at arrival $=$ RLS score of 4 to 8 ; unconscious or lethargic $=$ RLS $>1$; symptom-toneedle time = time between the onset of stroke symptoms and initiation of thrombolysis; and IVT = IVT with recombinant tissue plasminogen activator. In variables for which number and percent are reported, $p$ values were obtained by $\chi^{2}$, whereas in variables for which median and IQR are reported, $p$ values were obtained by Mann-Whitney test.

Arrival by ambulance: data are presented for years 2011 through 2014.

${ }^{a}$ The $p$ values for differences between people with and without dementia.

${ }^{b}$ Variables with missing data, $\mathrm{n}(\%)$ : nursing home placement, 42 (0.5); lives alone (not in a nursing home), 51 (0.6); independent (in mobility, dressing, and toilet visits), 192 (2.4); smoking, 712 (8.8); stroke code, 144 (1.8); arrival by ambulance, 654 (8.9) (for years 2011-2014); unconscious at arrival and unconscious or lethargic, 95 (1.2); and thrombolysis, 37 (0.5). 
Table 2 Demographics, medications, and comorbidities before stroke in patients with AIS treated with IVT

Demographics, medications, and comorbidities before

stroke

Age at first stroke, median (IQR), y

\section{Female sex, $\mathrm{n}(\%)$ \\ Nursing home placement, n (\%)}

Lives alone (not in a nursing home), $n(\%)^{a}$

ADL independent, $\mathrm{n}(\%)^{\mathrm{a}}$

Smoking, $n(\%)^{a}$

Medications, median (IQR), n

Antiaggregants, $\mathrm{n}(\%)$

Antipsychotics, n (\%)

Antidepressants, n (\%)

Antihypertensives, n (\%)

Antidiabetics, n (\%)

Statins, n (\%)

AF, $n(\%)$

Warfarin in patients with

AF, n (\%)

Diabetes mellitus, $\mathrm{n}$ (\%)

Hypertension, n (\%)

Femur fracture, $n$ (\%)

Any hemorrhage, $\mathrm{n}$ (\%)

Ischemic heart disease, n (\%)

Heart failure, n (\%)

Renal disease, $\mathrm{n}$ (\%)

Liver disease, n (\%)

Stroke code, n (\%)

Arrival by ambulance, $\mathrm{n}(\%)^{\mathrm{a}}$

Unconscious at arrival (RLS

4-8), n (\%)

Unconscious or lethargic

(RLS>1), n (\%) ${ }^{a}$

Symptom-to-needle time,

$\mathrm{n}(\%)^{\mathrm{a}}$

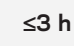

$$
>3-\leq 4.5 \mathrm{~h}
$$$$
>4.5 \mathrm{~h}
$$

NIHSS points before IVT, mean $\pm S D^{a}$

NIHSS points after IVT, mean $\pm S D^{a}$

NIHSS change, mean $\pm S D^{a}$

sICHa $^{a}$

In-hospital death

mRS score at $3 \mathrm{mo}^{\mathrm{a}}$

0-2

4 (4.9)

\begin{tabular}{|c|c|c|}
\hline $\begin{array}{l}\text { Patients with } \\
\text { dementia }(n=94)\end{array}$ & $\begin{array}{l}\text { Patients without } \\
\text { dementia }(n=639)\end{array}$ & p Value \\
\hline $83(6)$ & 81 (9) & 0.016 \\
\hline $54(57.4)$ & 353 (55.2) & 0.688 \\
\hline 29 (30.9) & $30(4.7)$ & $<0.001$ \\
\hline 24 (25.5) & 226 (35.5) & 0.058 \\
\hline 60 (65.2) & 597 (94.0) & $<0.001$ \\
\hline $8(9.0)$ & 43 (7.3) & 0.577 \\
\hline $6(4)$ & $4(4)$ & 0.001 \\
\hline 52 (55.3) & $259(40.5)$ & 0.007 \\
\hline 3 (3.2) & 8 (1.3) & $0.157^{b}$ \\
\hline 32 (34.0) & 68 (10.6) & $<0.001$ \\
\hline 63 (67.0) & 419 (65.6) & 0.782 \\
\hline 13 (13.8) & 74 (11.6) & 0.529 \\
\hline 21 (22.3) & $143(22.4)$ & 0.993 \\
\hline 31 (33.0) & 168 (26.3) & 0.173 \\
\hline 1 (3.2) & $17(10.1)$ & $0.318^{b}$ \\
\hline 15 (16.0) & 98 (15.3) & 0.876 \\
\hline 55 (58.5) & 316 (49.5) & 0.101 \\
\hline $5(5.3)$ & $36(5.6)$ & 0.901 \\
\hline $8(8.5)$ & 54 (8.5) & 0.984 \\
\hline 36 (38.3) & $175(27.4)$ & 0.029 \\
\hline 21 (22.3) & 104 (16.3) & 0.144 \\
\hline $4(4.3)$ & $47(7.4)$ & 0.27 \\
\hline 3 (3.2) & 8 (1.3) & $0.157^{b}$ \\
\hline 92 (97.9) & 615 (97.3) & 0.75 \\
\hline 86 (100) & 547 (94.6) & 0.028 \\
\hline 1 (1.1) & $9(1.4)$ & $1.0 \mathrm{~b}$ \\
\hline \multirow[t]{2}{*}{20 (21.3) } & 107 (16.9) & 0.291 \\
\hline & & $0.964^{b}$ \\
\hline
\end{tabular}

70 (80.5)

471 (80.8)

100 (17.2)

12 (2.1)

$10.0 \pm 6.4$

0.009

$6.3 \pm 7.4$

0.284

$-3.4 \pm 6.0 \quad 0.305$

46 (7.3)

85 (13.3)

0.960

0.886
7 (7.4)

12 (12.8) groups in the geographic county of origin or urban and rural groups (results not presented).

Symptom-to-needle time did not differ between patients with and those without dementia (table 2). Patients with dementia had a higher NIHSS score before IVT (12 vs 10 points, $p=0.009$ ). This difference in NIHSS score was no longer present in the evaluation after IVT treatment ( $p=0.284)$; however, the proportion of missing data was $29.3 \%$. There were no differences in $\mathrm{sICH}$, in-hospital death, and death at the 3-month follow-up (table 2), but 56.1\% of patients with dementia (compared to $18.8 \%$ of controls) presented with an mRS score of 5 or 6 at 3 months. Thrombectomy was performed in 3 (3.2\%) patients with dementia and $37(5.8 \%)$ patients without dementia receiving IVT $(p=0.307)$ (results not presented).

ORs for patients with dementia receiving IVT are presented in table 3 . In the fully adjusted covariate model (model 2) and in the post hoc propensity score-adjusted model (model 3), dementia was associated with lower odds of receiving thrombolysis. However, when the analysis was repeated exclusively among patients who were ADL independent before stroke, the difference between patients with and without dementia was no longer significant for the whole cohort (OR 0.79, 95\% CI 0.60-1.06), although differences persisted for patients $\leq 80$ years of age (table 3).

Thrombolysis outcomes. In the fully adjusted model, there were no differences in OR for death at the 3-month follow-up between patients with and those without dementia who received IVT. Functional outcome at 3 months, assessed with mRS, was worse among patients with dementia. In the fully adjusted covariate model, the OR for a higher mRS score in dementia was 3.65 (95\% CI 2.06-6.45). In patients with dementia, OR for new nursing home placement was tripled (table 4).

DISCUSSION In this large, nationwide, longitudinal study, we observed the following key findings: (1) patients with dementia were less likely to receive IVT, but these differences disappeared in analyses focusing on previously independent patients, persisting only in patients $\leq 80$ years of age; (2) the frequencies of sICH and in-hospital and 3-month mortality after IVT were similar in patients with dementia and controls; and (3) among patients who received IVT, dementia was associated with greater disability and new nursing home placement.

Before stroke, patients with preexisting dementia had more comorbidities and were more ADL dependent, which is in line with previous studies from SveDem. ${ }^{21}$ The proportion of IVT-treated patients (9.5\% for those without dementia and $7.0 \%$ for 


\section{Table 2 Continued}

Demographics, medications and comorbidities before stroke

3

4

5

(Dead) 6

New nursing home placement, $\mathrm{n}(\%)^{\mathrm{a}}$

$\begin{array}{llr}\begin{array}{l}\text { Patients with } \\ \text { dementia }(\mathbf{n}=94)\end{array} & \begin{array}{l}\text { Patients without } \\ \text { dementia }(\mathbf{n}=639)\end{array} & \text { p Value } \\ 20(24.4) & 155(27.2) & 0.592 \\ 12(14.6) & 66(11.6) & 0.425 \\ 28(34.1) & 53(9.3) & <0.001 \\ 18(22.0) & 107(18.8) & 0.494 \\ 23(35.9) & 64(13.6) & <0.001\end{array}$

Abbreviations: $A D L=$ activities of daily living; $A F=$ Atrial fibrillation; $A I S=$ acute ischemic stroke; IQR = interquartile range; IVT = intravenous thrombolysis; $\mathrm{mRS}=$ modified Rankin Scale; NIHSS = NIH Stroke Scale; RLS = Reaction Level Scale; sICH = symptomatic intracranial hemorrhage.

Symptom-to-needle time is time between the onset of stroke symptoms and initiation of thrombolysis. IVT is with recombinant tissue plasminogen activator.

In variables for which number and percent are reported, $p$ values were obtained by $\chi^{2}$. In variables for which median with IQR are reported, $p$ values were obtained by the MannWhitney test. In variables for mean and SD are reported, $p$ values were obtained by Student $t$ test.

Arrival by ambulance: data are presented for years 2011 through 2014. New nursing home placement: for survivors of hospitalization (82 patients with dementia and 554 patients without dementia).

a Variables with missing data, $\mathrm{n}$ (\%): lives alone (not in a nursing home), 2 (0.3); ADL independent (in mobility, dressing, and toilet visits), 6 (0.8); smoking, 56 (7.6); stroke code, 7 (1.0); arrival by ambulance, 29 (4.2) (for years 2011-2014); unconscious at arrival and unconscious or lethargic at arrival, 4 (0.5); symptom-to-needle time, 63 (8.6); NIHSS points before IVT, 60 (8.2); sICH, 9 (1.2); mRS at 3 months, 81 (11.1); and new nursing home placement, 103 (16.2).

${ }^{\mathrm{b}}$ Fisher exact test.
Worse functional prognosis after stroke in older patients ${ }^{24}$ and in those with dementia ${ }^{7,8}$ could lead to therapeutic nihilism and withholding of treatment. On the other hand, excessive enthusiasm for IVT could expose individuals to serious complications. Prior studies on IVT in AIS and dementia investigated death and $\mathrm{sICH},{ }^{7-9}$ and to the best of our knowledge, only one study specifically addressed functional outcomes after IVT. ${ }^{10}$ In that study, the proportion of independent patients with dementia in the initial cohort $(28.9 \%)$ and in the cohort who received IVT (48.5\%) was lower compared to ours ( $52.8 \%$ and $65.2 \%$, respectively). This may be due to different definitions of independence or to the fact that dementia diagnosis in their study originated from clinical records (possibly including later dementia stages), whereas we included patients at the time of dementia diagnosis. ${ }^{11}$ In SveDem, $>60 \%$ of patients are diagnosed with dementia with an MMSE score of $\geq 20 .{ }^{12}$ In our study, patients with dementia received the same standard of care regarding transport by ambulance and speedy initiation of IVT, but younger patients with dementia were less likely to have an activation of stroke code.

In line with previous studies, ${ }^{25}$ patients with dementia and AIS who received IVT were more selected, which is supported by the lower IVT rate in this group compared to controls. It seems that the decision criteria in both patients with and without dementia were functional status and age, because the difference could not be attributed solely to greater comorbidities and resultant contraindications, for which we adjusted. However, there might still be some residual confounding for which we could not account. Current American guidelines for IVT in dementia are subject to individual judgment, and they suggest taking into account life expectancy, premorbid functional level, and clinically meaningful

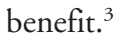
guidelines. ${ }^{3,19}$

patients with dementia) was slightly higher than other recent national averages $(6.4 \%$ in New Zealand in $2016,{ }^{22} 6.1 \%$ in the United States $\left.{ }^{23}\right)$.

Patients treated with IVT (those with dementia and controls) were more independent than their counterparts who did not receive IVT. Moreover, ADL independence acted as a mediator for receiving IVT; hence, it probably represents an important decision factor for a physician contemplating IVT treatment, which is in line with the current

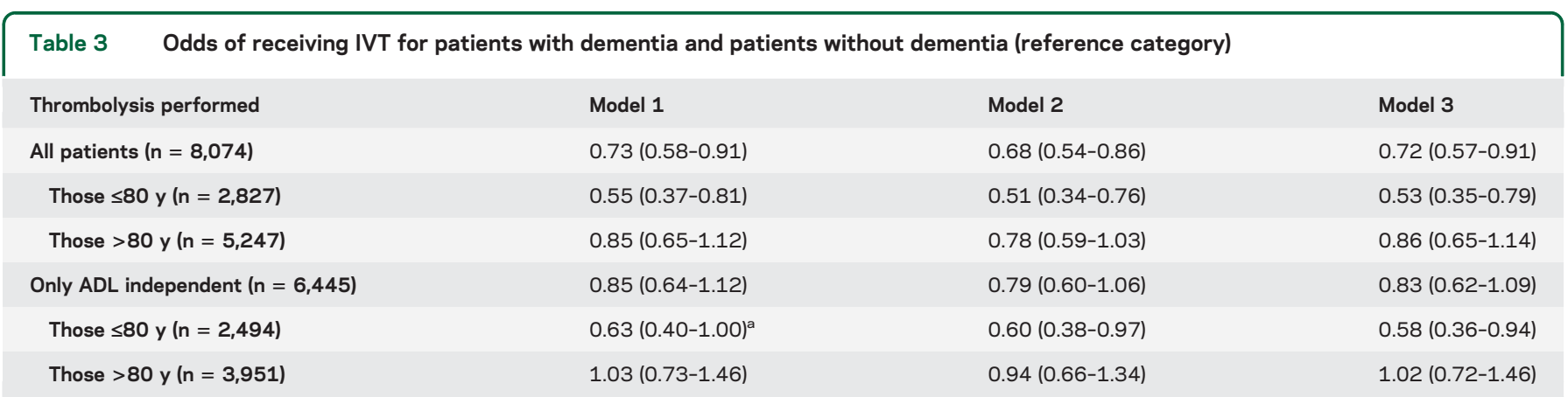

Abbreviations: $\mathrm{ADL}=$ activities of daily living; IVT $=$ intravenous thrombolysis.

Results are derived from logistic regression analysis for association between receiving thrombolysis and dementia status. Data are presented as odds ratio with $95 \%$ confidence interval. Model 1 is adjusted for age and sex. Model 2 is adjusted for age, sex, living alone, unconscious state at arrival, femur fracture, warfarin, interaction between heart failure and antiaggregants, and number of drugs without antithrombotics. Model 3 is adjusted for propensity scores of dementia.

${ }^{a} p=0.051$ 
Table 4 Odds for mRS, death, and accommodation at 3 months after intravenous thrombolysis for patients with and without dementia (reference category)

\begin{tabular}{|c|c|c|c|c|}
\hline Outcomes of thrombolysis & Model 1 & Model 2 & Model 3 & Model 4 \\
\hline mRS score at 3 months & 5.63 (3.41-9.30) & $3.49(2.02-6.01)$ & $3.65(2.06-6.45)$ & $4.67(2.81-7.75)$ \\
\hline$m R S$ score of 4 or 5 & $4.48(2.56-7.84)$ & $2.74(1.48-5.05)$ & $2.47(1.21-5.05)$ & $3.72(2.10-6.59)$ \\
\hline Death at 3 months & $1.06(0.60-1.90)$ & $0.83(0.44-1.53)$ & $0.71(0.36-1.8)$ & $1.03(0.58-1.84)$ \\
\hline New nursing home placement & 3.19 (1.78-5.72) & 3.83 (1.98-7.42) & 4.39 (2.07-9.31) & 3.29 (1.79-6.07) \\
\hline
\end{tabular}

Abbreviation: $\mathrm{mRS}=$ modified Rankin Scale.

Results are presented as odds ratio with $95 \%$ confidence interval.

For mRS score at 3 months, results are derived from ordered logistic regression analysis. Model 1 was adjusted for age and sex, and the analysis was conducted on 527 patients. Model 2 was adjusted for age, sex, nursing home placement, antidepressants, and number of drugs minus antidepressants. Model 3 was adjusted as model 2 but also included NIH Stroke Scale score before thrombolysis (post hoc analysis).

For mRS score of 4 or 5 at 3 months, results are derived from multiple logistic regression analyses, reporting odds ratio of mRS score of 4 or 5 (vs 0 to 3 ) at 3 months, and are adjusted as above.

For death at 3 months, results are derived from multiple logistic regression analysis. Model 1 was adjusted for age and sex, while model 2 was adjusted for age, sex, nursing home placement, and number of medication. Analysis was conducted on all patients who received thrombolysis ( $\mathrm{n}=733$ ). Model 3 included also NIH Stroke Scale score before thrombolysis (post hoc analysis).

For new nursing home placement, results are derived from multiple logistic regression analysis. Analysis was conducted on 529 patients. Model 1 was adjusted for age and sex. Model 2 was adjusted for age, sex, living alone (not in a nursing home), prior independence in activities of daily living, and number of medication. Model 3 included also NIH Stroke Scale score (post hoc analysis).

All results for model 4 were adjusted with propensity scores (post hoc analysis).

Some studies found that mortality after stroke in dementia is increased regardless of the use of IVT, ${ }^{7,8}$ which could be partly explained by worse baseline status. ${ }^{26}$ A recent SveDem study showed that stroke was a substantial cause of death in dementia. ${ }^{27}$ The present study did not find differences in mortality in IVT-treated patients with dementia during hospitalization or at the 3month follow-up, but functional outcomes were worse. The incidence of sICH was similar $(7.4 \%$ vs $7.3 \%, p=0.960)$.

We found no differences between the 2 groups in improvement in NIHSS score, but the proportion of missing data was high $(\approx 30 \%)$. The initial difference in NIHSS could not be explained by the degree of consciousness, which was similar between groups, but might be due to greater stroke severity, confusional state, or poorer understanding of instructions in patients with both dementia and AIS.

IVT-treated patients with dementia had worse functioning at the 3-month follow-up. In a study from $2012,{ }^{10}$ dementia itself was not an independent predictor of a worse functional outcome. However, the groups were matched for prior residence and preadmission dependency, whereas we adjusted only for residence. Besides dementia, factors associated with poor outcome in our model were age, prior nursing home placement, and total number of medication. Consistent with a previous study based in the United States, ${ }^{7}$ our IVT-treated patients with dementia had an unfavorable discharge destination compared to controls. In our study, dementia was independently associated with a new nursing home placement, a finding that differs from previous studies. ${ }^{10}$ The odds for patients with dementia of being placed in a long-term facility were increased (OR 3.83, 95\% CI $1.98-7.42)$.

A multicenter European study investigated the effects of preexisting dependency (often dementia) on outcomes and complications of IVT. ${ }^{26}$ Dependent patients presented higher mortality rates; however, in our study, the incidence of poor outcome in survivors did not differ. Thus, previously dependent patients might still benefit from IVT.

This study has several limitations. First, baseline functioning in our cohort could be assessed only indirectly from degree of independence and living situation, while only an mRS estimation was available for outcome. Other methods of measuring functioning could be of interest, but the study could include only those assessed in Riksstroke. Second, the percentage of missing NIHSS after thrombolysis was $\approx 30 \%$, limiting its usefulness. Third, nursing home residents with dementia and AIS are probably underrepresented in this study because they may be less often referred to hospitals. Causal inference is limited in cohort studies. IVT is an established treatment with a known risk-benefit profile. Our study shows that $\mathrm{sICH}$ and mortality outcomes were similar between patients with dementia and patients without dementia, although functional outcomes were worse. This may assuage some concerns when IVT is administered to this specific population. Those with dementia were eliminated from the control group by the exclusion of patients who were ever registered in SveDem, received a dementia or confusion diagnosis in the Swedish National Inpatient Register, or took antidementia drugs, but perfect case ascertainment is impossible. Riksstroke offers a $>90 \%$ national coverage of ischemic stroke events, and the national 
dementia registry, SveDem, provides superior accuracy in dementia diagnoses compared to claims data. However, in 2012, the estimated coverage of incident dementia cases in SveDem was 36\%, ${ }^{11}$ and the data on cognitive status (MMSE) were obtained at the time of dementia diagnosis, a median of 1.5 years before the stroke. SveDem may not be representative of the general dementia population, and dementia severity at the time of stroke is impossible to ascertain. SveDem diagnoses are not externally validated, but only $\approx 5 \%$ of patients change diagnoses at follow-up, suggesting that the initial diagnoses are robust. ${ }^{12} \mathrm{~Pa}$ tients may refuse participation in SveDem or Riksstroke, and no information is collected on nonincluded patients. However, inclusion is the default, and refusal is generally low in our clinical experience. The absence of dementia among the control group was ascertained through SveDem and other registries, but further exclusion of dementia cases (by examining patients or their journals) was not attempted and is an important limitation of this study.

To the best of our knowledge, there have been no studies on this topic since the prolongation of the IVT treatment window to 4.5 hours in 2009 , and we are the first to report symptom-to-needle time in this group of patients. The large nationwide cohort, detailed dementia and stroke characterization, and low proportion of missing (including for the 3-month follow-up) are strengths of our study.

The present study, investigating use of IVT and its outcomes in dementia, suggests that in selected patients IVT is safe, with rates of treatment complications similar to those in patients without dementia. Patients with dementia have worse functional outcomes after IVT, which might be explained by worse baseline functional status.

\section{AUTHOR CONTRIBUTIONS}

E. Zupanic: study concept, drafting the manuscript, statistical analysis and interpretation of data. M. von Euler: study concept, acquisition of data, and revising the manuscript for scientific content. I. Kåreholt: study concept, statistical analysis and interpretation of data, revising the manuscript. B. Contreras Escamez, Johan Fastbom, and Bo Norrving: interpretation of data and revising the manuscript. Dorota Religa: study concept, interpretation of data, and revising the manuscript. Milica G. Kramberger, Bengt Winblad, and Kristina Johnell: interpretation of data and revising the manuscript. Maria Eriksdotter: study concept and design, data acquisition, interpretation of data and revising the manuscript. Sara Garcia-Ptacek: study concept and design, data acquisition, statistical analysis and interpretation of data, and drafting and revising the manuscript.

\section{ACKNOWLEDGMENT}

The authors thank all patients, caregivers, reporting units, and coordinators in SveDem and Riksstroke, as well as the SveDem and Riksstroke steering committees. The authors are grateful to Marie Eriksson (Riksstroke) for her help with database variables.

\section{STUDY FUNDING}

This project was conducted with support from the Swedish Order of Saint John/Johanniterorden i Sverige, the Swedish Society for Medical
Research, the Swedish Stroke Association, Stiftelsen Dementia, the Swedish Research Council, and the Swedish Associations of Local Authorities and Regions. D. Religa was supported by a Swedish Research Council grant (2012-2291).

\section{DISCLOSURE}

E. Zupanic, M. von Euler, I. Kåreholt, and B. Contreras Escamez report no disclosures relevant to the manuscript. J. Fastbom is funded by the Swedish Research Council for Health, Working Life and Welfare. He is associate editor for the European Journal of Clinical Pharmacology. B. Norrving is a member of the Data Monitoring Committee for the Rivaroxaban Versus Aspirin in Secondary Prevention of Stroke and Prevention of Systemic Embolism in Patients With Recent Embolic Stroke of Undetermined Source (ESUS) NAVIGATE-ESUS trial, has been a member of the Data Monitoring Committee of the Acute Stroke or Transient Ischaemic Attack Treated With Aspirin or Ticagrelor and Patient Outcomes (SOCRATES) trial, is editor-in-chief for the European Stroke Journal and associate editor for Neuroepidemiology, and has served as senior consulting editor for Stroke. D. Religa and M. Kramberger report no disclosures relevant to the manuscript. B. Winblad is funded by the Swedish Research Council, Brain Foundation, Old Servants' Foundation, and Stohne's Stiftelsen and serves on Scientific Advisory Boards for AC Immune, Axon Pharma, Axovant, Janssen, Merck, and Novartis. K. Johnell reports no disclosures relevant to the manuscript. M. Eriksdotter is funded by the Swedish Research Council, The Brain Foundation, Swedish Order of Saint John, and Swedish Associations of Local Authorities and Regions. She has received honoraria for moderating an event on advanced dementia by the Swedish medical association journal and for lectures on the Norwegian dementia days. S. Garcia-Ptacek is funded by the Swedish Order of Saint John/Johanniterorden and the Swedish Society for Medical Research. Go to Neurology.org for full disclosures.

Received March 29, 2017. Accepted in final form August 11, 2017.

\section{REFERENCES}

1. Lees KR, Bluhmki E, Von Kummer R, et al. Time to treatment with intravenous alteplase and outcome in stroke: an updated pooled analysis of ECASS, ATLANTIS, NINDS, and EPITHET trials. Lancet 2010;375:1695-1703.

2. Subic A, Cermakova P, Norrving B, et al. Management of acute ischaemic stroke in patients with dementia. J Intern Med 2017;281:348-364.

3. Demaerschalk BM, Kleindorfer DO, Adeoye OM, et al. Scientific rationale for the inclusion and exclusion criteria for intravenous alteplase in acute ischemic stroke: a statement for healthcare professionals from the American Heart Association/American Stroke Association. Stroke 2016;47: 581-641.

4. European Medicines Agency. Summary Information on a Referral Opinion Following an Arbitration Pursuant to Article 29 of Directive 2001/83/EC, for Actilyse [online]. Available at: http://www.ema.europa.eu/docs/en_GB/document_ library/Referrals_document/Actilyse_29/WC500010327.pdf. Accessed June 16, 2017.

5. European Stroke Organisation. Statement on Revised Guidelines for Intravenous Thrombolysis [online]. Available at: http://www.congrex-switzerland.com/fileadmin/files/2013/esostroke/pdf/ESO_Guideline_Update_Jan_2009.pdf. Accessed June 16, 2017.

6. Paciaroni M, Pantoni L. Thrombolysis in dementia patients with acute stroke: is it justified? Neurol Sci 2016; 38:27-31.

7. Busl KM, Nogueira RG, Yoo AJ, Hirsch JA, Schwamm LH, Rost NS. Prestroke dementia is associated with poor outcomes after reperfusion therapy among elderly stroke patients. J Stroke Cerebrovasc Dis 2013;22:718-724. 
8. Saposnik G, Cote R, Rochon PA, et al. Care and outcomes in patients with ischemic stroke with and without preexisting dementia. Neurology 2011;77:1664-1673.

9. Alshekhlee A, Li CC, Chuang SY, et al. Does dementia increase risk of thrombolysis? A case-control study. Neurology 2011;76:1575-1580.

10. Saposnik G, Kapral MK, Cote R, et al. Is pre-existing dementia an independent predictor of outcome after stroke? A propensity score-matched analysis. J Neurol 2012;259:2366-2375.

11. Religa D, Fereshtehnejad SM, Cermakova P, et al. SveDem, the Swedish Dementia Registry: a tool for improving the quality of diagnostics, treatment and care of dementia patients in clinical practice. PLoS One 2015; 10:e0116538.

12. Garcia-Ptacek S, Farahmand B, Kareholt I, Religa D, Cuadrado ML, Eriksdotter M. Mortality risk after dementia diagnosis by dementia type and underlying factors: a cohort of 15,209 patients based on the Swedish Dementia Registry. J Alzheimers Dis 2014;41:467-477.

13. Asplund K, Hulter Asberg K, Norrving B, et al. Riksstroke: a Swedish national quality register for stroke care. Cerebrovasc Dis 2003;15(suppl 1):5-7.

14. Asplund K, Sukhova M, Wester P, Stegmayr B. Diagnostic procedures, treatments, and outcomes in stroke patients admitted to different types of hospitals. Stroke 2015;46: 806-812.

15. Wettermark B, Hammar N, MichaelFored C, et al. The new Swedish Prescribed Drug Register: opportunities for pharmacoepidemiological research and experience from the first six months. Pharmacoepidemiol Drug Saf 2007; 16:726-735.

16. Ludvigsson JF, Andersson E, Ekbom A, et al. External review and validation of the Swedish National Inpatient Register. BMC Public Health 2011;11:450.

17. Eurostat Statistics Explained: Urban-Rural Typology [online]. Available at: http://ec.europa.eu/eurostat/statistics-explained/ index.php/Urban-rural_typology. Accessed February 20, 2017.

18. Eriksson M, Appelros P, Norrving B, Terént A, Stegmayr B. Assessment of functional outcome in a national quality register for acute stroke: can simple self-reported items be transformed into the modified Rankin Scale? Stroke 2007; 38:1384-1386.

19. Socialstyrelsen. National Guidelines for Stroke Care (Nationella riktlinjer för strokesjukvård) [online in Swedish]. 2009. Available at: http://www.socialstyrelsen.se/Lists/ Artikelkatalog/Attachments/17790/2009-11-4.pdf. Accessed January 10, 2017.

20. Garcia-Ptacek S, Kareholt I, Farahmand B, Cuadrado ML, Religa D, Eriksdotter M. Body-mass index and mortality in incident dementia: a cohort study on 11,398 patients from SveDem, the Swedish Dementia Registry. J Am Med Dir Assoc 2014;15:447.e1-447.e7.

21. Cermakova P, Johnell K, Fastbom J, et al. Cardiovascular diseases in $\sim 30,000$ patients in the Swedish Dementia Registry. J Alzheimers Dis 2015;48:949-958.

22. Joshi P, Fink J, Barber PA, et al. Stroke thrombolysis in New Zealand: data from the first 6 months of the New Zealand Thrombolysis Register. N Z Med J 2016; 129:44-49.

23. Kim JT, Fonarow GC, Smith EE, et al. Treatment with tissue plasminogen activator in the golden hour and the shape of the 4.5-hour time-benefit curve in the national United States Get With The Guidelines-Stroke population. Circulation 2017;135:128-139.

24. Engelter ST, Bonati LH, Lyrer PA. Intravenous thrombolysis in stroke patients of $>$ or $=80$ versus $<80$ years of age: a systematic review across cohort studies. Age Ageing 2006;35:572-580.

25. Karlinski M, Kobayashi A, Czlonkowska A, et al. Role of preexisting disability in patients treated with intravenous thrombolysis for ischemic stroke. Stroke 2014; 45:770-775.

26. Gensicke H, Strbian D, Zinkstok SM, et al. Intravenous thrombolysis in patients dependent on the daily help of others before stroke. Stroke 2016;47:450-456.

27. Garcia-Ptacek S, Kareholt I, Cermakova P, Rizzuto D, Religa D, Eriksdotter M. Causes of death according to death certificates in individuals with dementia: a cohort from the Swedish Dementia Registry. J Am Geriatr Soc 2016;64:e137-e142.

\section{Share Your Artistic Expressions in Neurology 'Visions'}

AAN members are urged to submit medically or scientifically related artistic images, such as photographs, photomicrographs, and paintings, to the "Visions" section of Neurology ${ }^{\circledR}$. These images are creative in nature, rather than the medically instructive images published in the NeuroImages section. The image or series of up to six images may be black and white or color and must fit into one published journal page. Accompanying description should be 100 words or less; the title should be a maximum of 96 characters including spaces and punctuation.

Learn more at www.aan.com/view/Visions, or upload a Visions submission at submit.neurology.org. 


\section{Neurology}

Thrombolysis in acute ischemic stroke in patients with dementia: A Swedish registry study

Eva Zupanic, Mia von Euler, Ingemar Kåreholt, et al.

Neurology 2017;89;1860-1868 Published Online before print October 6, 2017

DOI 10.1212/WNL.0000000000004598

This information is current as of October 6, 2017

Neurology ${ }^{\circledR}$ is the official journal of the American Academy of Neurology. Published continuously since 1951, it is now a weekly with 48 issues per year. Copyright Copyright (C) 2017 The Author(s). Published by Wolters Kluwer Health, Inc. on behalf of the American Academy of Neurology. All rights reserved. Print ISSN: 0028-3878. Online ISSN: 1526-632X.

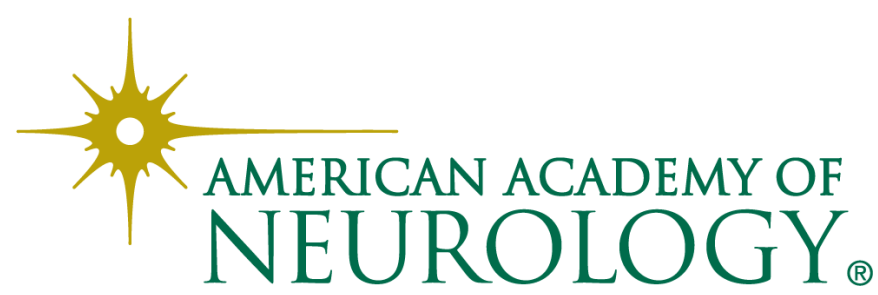




\section{Updated Information \& Services}

References

Citations

Subspecialty Collections

\section{Errata}

Permissions \& Licensing

Reprints including high resolution figures, can be found at: http://n.neurology.org/content/89/18/1860.full

This article cites 23 articles, 8 of which you can access for free at: http://n.neurology.org/content/89/18/1860.full\#ref-list-1

This article has been cited by 1 HighWire-hosted articles: http://n.neurology.org/content/89/18/1860.full\#\#otherarticles

This article, along with others on similar topics, appears in the following collection(s):

All Cognitive Disorders/Dementia

http://n.neurology.org/cgi/collection/all_cognitive_disorders_dementia All global neurology

http://n.neurology.org/cgi/collection/all_global_neurology

Burden of disease

http://n.neurology.org/cgi/collection/burden_of_disease

Cohort studies

http://n.neurology.org/cgi/collection/cohort_studies

Infarction

http://n.neurology.org/cgi/collection/infarction

Prognosis

http://n.neurology.org/cgi/collection/prognosis

Public health

http://n.neurology.org/cgi/collection/public_health

An erratum has been published regarding this article. Please see next page or:

/content/97/3/151.2.full.pdf

Information about reproducing this article in parts (figures,tables) or in its entirety can be found online at:

http://www.neurology.org/about/about_the_journal\#permissions

Information about ordering reprints can be found online:

http://n.neurology.org/subscribers/advertise

Neurology ${ }^{\circledR}$ is the official journal of the American Academy of Neurology. Published continuously since 1951, it is now a weekly with 48 issues per year. Copyright Copyright ( 2017 The Author(s). Published by Wolters Kluwer Health, Inc. on behalf of the American Academy of Neurology. All rights reserved. Print ISSN: 0028-3878. Online ISSN: 1526-632X.

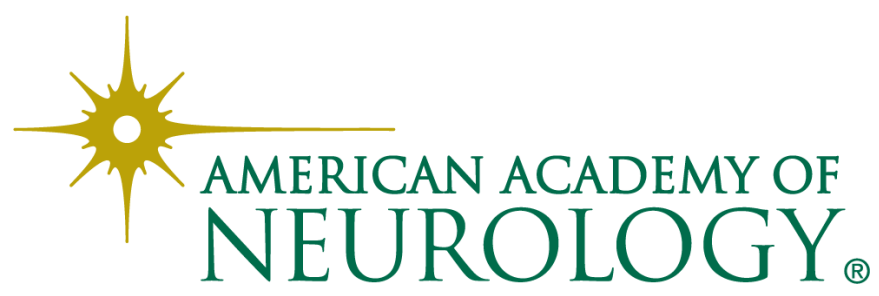




\title{
Disputes \& Debates: Editors' Choice
}

Steven Galetta, MD, FAAN, Editor

Aravind Ganesh, MD, DPhil, FRCPC, Deputy Editor

Ariane Lewis, MD, Deputy Editor

James E. Siegler III, MD, Deputy Editor

\section{Editors' Note: Cause of Death in Spontaneous Intracerebral Hemorrhage Survivors: Multistate Longitudinal Study}

Using administrative claims data from 3 US states, Dr. Kuohn et al. report the long-term mortality rate of survivors with spontaneous intracerebral hemorrhage (ICH). Using an original population of 104,000 patients who were screened for eligibility, 72,432 survivors of the index hospitalization were followed for a median of 4 years. The 1-year risk of death or discharge to hospice was $9.5 \%$ (95\% CI 9.2\%-9.7\%), with the most common proximate causes of death being infection (34\%) or subsequent intracranial hemorrhage (13\%). The median time from ICH to death was 10 months (IQR 2-31 months). Dr. Llamas-Nieves and colleagues highlight the importance of confounders such as diabetes, which was independently associated with death as shown in the multivariable regression model (HR $1.22,95 \%$ CI 1.17-1.27), as this may be an important mediator of death because of infectious causes. The investigators acknowledge that diabetes contributes to death in the overall population; however, it was not independently associated with death due to infection (OR 1.16, 95\% CI 0.86-1.55). The authors posit that diabetes (as well as age, postICH disability, and other comorbidities) are important contributors to the development of later infection, but it remains unclear why diabetes is not an independent modifier of death risk among patients who develop infection.

James E. Siegler, MD, and Steven Galetta, MD

Neurology ${ }^{\circledR}$ 2021;97:148. doi:10.1212/WNL.0000000000012281

\section{Reader Response: Cause of Death in Spontaneous Intracerebral Hemorrhage Survivors: Multistate Longitudinal Study}

\author{
Andrés E. Llamas-Nieves (Cartagena, Colombia), Ivan D. Lozada-Martinez (Cartagena, Colombia), \\ Daniela M. Torres-Llinás (Cartagena, Colombia), and Luis R. Moscote-Salazar (Cartagena, Colombia) \\ Neurology ${ }^{\circledR}$ 2021;97:148-149. doi:10.1212/WNL.0000000000012282
}

We read with great interest the recent article by Kuohn et al. ${ }^{1}$ The authors conducted a comprehensive review on the factors associated with mortality in patients who survived spontaneous intracerebral hemorrhage, where they showed that the infection was the main cause of death in such a group with a value of $34 \%$. However, they do not emphasize other comorbidities that could have influenced the morbidity and mortality of patients in the infection group, such as diabetes. Diabetes is a disease that increases susceptibility to infections such as those involving the skin, urinary tract, or lungs. ${ }^{2}$ Lau et al. performed a systematic review and meta-analysis, where they found that isolated diabetes is strongly associated with poor prognosis after stroke, including increased morbidity, mortality, and disability. ${ }^{3}$ For this reason, it is very important to know the factors that underlie and really impact on the subject of study, to obtain certainty in the 
results of the research, and to avoid underestimations as what likely happened in the study of Kuohn et al. We suggest separate future study groups to avoid this type of bias during data analysis.

1. Kuohn LR, Leasure AC, Acosta JN, et al. Cause of death in spontaneous intracerebral hemorrhage survivors Multistate longitudinal study. Neurology. 2020;95(20):e2736-e2745.

2. Pannu AK, Saroch A, Singla V, et al. Clinical spectrum, etiology and outcome of infectious disease emergencies in adult diabetic patients in northern India. Diabetes Metab Syndr. 2020;14(5):921-925.

3. Lau L-H, Lew J, Borschmann K, Thijs V, Ekinci EI. Prevalence of diabetes and its effects on stroke outcomes: a meta-analysis and literature review. J Diabetes Investig. 2019;10(3):780-792.

Copyright (c) 2021 American Academy of Neurology

\section{Author Response: Cause of Death in Spontaneous Intracerebral Hemorrhage Survivors: Multistate Longitudinal Study}

Lindsey Kuohn (New York) and Kevin Sheth (New Haven, CT)

Neurology ${ }^{\circledR}$ 2021;97:149. doi:10.1212/WNL.0000000000012283

We thank Dr. Llamas-Nieves et al. for their interest in our article ${ }^{1}$ and recognize that coexisting diabetes, among other comorbidities, may influence infection-related mortality in ICH survivors. We hypothesized that the high proportion of deaths caused by infections may be due to the burden of disability in this population. Intracerebral hemorrhage ( $\mathrm{ICH}$ ) survivors often require chronic hospitalization and are prone to infections such as Clostridium difficile, urinary tract infections, and aspiration pneumonia. Our finding that older age was an independent risk factor for death caused by infection supports this hypothesis. However, we agree that increased susceptibility to infection in patients with diabetes should be considered as a potential driver of infection-related deaths in patients with $\mathrm{ICH}^{2}$ Diabetes was included as a covariate in the adjusted multinomial logistic regression model of causes of death during follow-up in our study (table 3). Diabetes was not associated with increased odds of death caused by infection (odds ratio 1.16, 95\% confidence interval 0.86-1.55), although diabetes did correlate with increased risk for death overall (hazard ratio $1.22,95 \%$ confidence interval 1.17-1.27). The cause of elevated mortality after ICH in patients with diabetes is not clear, and our results are not conclusive regarding the potential association with infection. As Dr. Llama-Nieves et al. point out, future studies of the specific causes of infection-related morbidity and mortality are warranted and necessary to improve long-term outcomes after ICH.

1. Kuohn LR, Leasure AC, Acosta JN, et al. Cause of death in spontaneous intracerebral hemorrhage survivors: multistate longitudinal study. Neurology. 2020;95(20):e2736-e2745.

2. Moutschen MP, Scheen AJ, Lefebvre PJ. Impaired immune responses in diabetes mellitus: analysis of the factors and mechanisms involved. Relevance to the increased susceptibility of diabetic patients to specific infections. Diabete Metab. 1992;18(3): $187-201$.

Copyright (c) 2021 American Academy of Neurology

Author disclosures are available upon request (journal@neurology.org). 


\section{Editors' Note: Sex, Race, and Risk of Dementia Diagnosis After Traumatic Brain Injury Among Older Veterans}

Traumatic brain injury (TBI) has a well-known association with cognitive impairment and dementia. Whether there is a differential risk of dementia after TBI based on sex, race, or ethnicity was the subject of the recently published retrospective cohort study by Dr. Kornblith et al. Using a $2 \%$ random sample of claims data from Veterans Health Administration systems databases $(n=999,640)$, investigators evaluated the cumulative incidence of all-cause dementia by age, sex, race, and ethnicity. They found a significant interaction between TBI and race for dementia, in which White veterans were at the highest risk for dementia diagnoses. Dr. Roberts affirms these findings are consistent with the literature on White vs Black NFL players, in which White athletes are diagnosed more frequently with postconcussion "dementia" and receive greater compensation for this disability. More importantly, Dr. Roberts emphasizes that this racial discrepancy in dementia diagnoses is not likely an indicator of differential disease burden between races but is more reflective of racial care inequality. Dr. Kornblith agrees that racial disparities in health care are ubiquitous and demand more than just our attention. They demand action.

James E. Siegler, MD, and Steven Galetta, MD

Neurology ${ }^{\circledR}$ 2021;97:150. doi:10.1212/WNL.0000000000012284

\section{Reader Response: Sex, Race, and Risk of Dementia Diagnosis After Traumatic Brain Injury Among Older Veterans}

Jomar S. Roberts I (Matthews, NC)

Neurology ${ }^{\circledR} 2021 ; 97: 150$. doi:10.1212/WNL.0000000000012288

I read the article by Kornblith et al. with interest. ${ }^{1}$ It seems this "trend" in diagnostic bias is not exclusive to veterans alone. As this article is being published, Black NFL and former NFL athletes are involved in litigation regarding the discrepancy in qualifying postconcussion dementia diagnoses applied to White athletes vs Black. White athletes are diagnosed with postconcussion dementia at higher rates and are thereby compensated for this at higher rates than Black athletes. ${ }^{2}$

In my experience as a veteran TBI neurologist, observing this bias is commonplace and not limited to concussion or any particular disease. The health care system is riddled with bias from the provider level on down. It manifests openly in less care being given to Black patients, less concern being given to their disease, and less resources allocated to deep diving into their illnesses. This is not a secret to Black neurologists or providers in any field and is openly discussed and hard fought against, daily.

To be perfectly honest, these authors are speaking softly about an issue that needs to be addressed much more openly. Racial bias in health care provision starts at the individual provider level. We need to further examine how providers' backgrounds affect their patterns of diagnosis, test ordering, and prescribing patterns. This should be prominently displayed in some metric for all patients to use when choosing a provider, a "bias report card" so to speak. Only with full transparency will patients be given the assurance their doctor/provider is giving full, ethically unbiased care to all.

1. Kornblith E, Peltz CB, Xia F, et al. Sex, race, and risk of dementia diagnosis after traumatic brain injury among older veterans. Neurology. 2020;95(13):e1768-e1775.

2. Radnofsky L, Beaton A. Lawsuit alleges NFL's concussion settlement discriminates against black players. In: The Wall Street Journal. Available at: wsj.com/articles/lawsuit-alleges-nfls-concussion-settlement-discriminates-against-black-players-11598371843. 


\section{Author Response: Sex, Race, and Risk of Dementia Diagnosis After Traumatic Brain Injury Among Older Veterans}

Erica S. Kornblith (San Francisco)

Neurology ${ }^{\circledR}$ 2021;97:151. doi:10.1212/WNL.0000000000012286

Thank you for the thoughtful comment on our article, ${ }^{1}$ Dr. Roberts. We agree that racial bias in health care is pervasive and must be addressed. We hope that our work draws attention to this issue and highlights the need for research aimed at identifying and mitigating disparities.

1. Kornblith E, Peltz CB, Xia F, et al. Sex, race, and risk of dementia diagnosis after traumatic brain injury among older veterans. Neurology. 2020;95(13):e1768-e1775.

Copyright @ 2021 American Academy of Neurology

\section{CORRECTIONS}

\section{Thrombolysis in Acute Ischemic Stroke in Patients With Dementia}

A Swedish Registry Study

Neurology ${ }^{\circledR}$ 2021;97:151. doi:10.1212/WNL.0000000000010325

In the article "Thrombolysis in Acute Ischemic Stroke in Patients With Dementia: A Swedish Registry Study" by Zupanic et al., ${ }^{1}$ the affiliations for Drs. Zupanic and Kramberger should include "Faculty of Medicine, University of Ljubljana, Slovenia." The authors regret the error.

\section{Reference}

1. Zupanic E, von Euler M, Kåreholt I, et al. Thrombolysis in acute ischemic stroke in patients with dementia: a Swedish registry study. Neurology. 2017;89(18):1860-1868.

\section{ALS is a Multistep Process in South Korean, Japanese, and Australian Patients}

Neurology ${ }^{\circledR}$ 2021;97:151. doi:10.1212/WNL.0000000000010323

In the infographic corresponding with the article "ALS is a Multistep Process in South Korean, Japanese, and Australian Patients" by Vucic et al., ${ }^{1}$ the labels for the pictures of South Korea and Japan should be switched so that South Korea reads as a 5-step process and Japan reads as a 6-step process. The editorial team regrets the error.

\section{Reference}

1. Vucic S, Higashihara M, Sobue G, et al. ALS is a multistep process in South Korean, Japanese, and Australian patients. Neurology. 2020; 94(15):e1657-e1663. 